\title{
Energy Budgeting for Battery-Powered Sensors with a Known Task Schedule
}

\author{
Daler Rakhmatov \\ ECE Department, University of Victoria \\ Victoria, British Columbia, CANADA \\ daler@uvic.ca
}

\begin{abstract}
Battery-powered wireless sensors are severely constrained by the amount of the available energy. A method for computing the energy budget per sensing task can be a valuable design aid for sensor network optimizations. This work presents such a method that computes the upper and lower bounds on the task energy budget for a sensor node that must remain operational over a specified lifetime, with a known task schedule. These bounds take into account nonlinear changes in the battery voltage, capacity loss at high discharge rates, charge recovery, and capacity fade over time. We also propose efficient approximations replacing expensive calculations of the battery voltage while computing the energy budget bounds.
\end{abstract}

\section{Categories and Subject Descriptors}

C.4 [Performance of Systems]: Performance attributes; J.6 [Computer-Aided Engineering]: Computer-aided design (CAD)

\section{General Terms}

Design, Performance

\section{Keywords}

Low-power design, battery voltage modeling, energy bounds

\section{INTRODUCTION}

Wireless sensor network systems, such as MIT's $\mu$ AMPS [7] and Intel/Berkeley's Motes [6], have been deployed for a wide spectrum of applications from environmental monitoring to object localization and tracking. Since sensor nodes are usually powered by batteries, efficient use of the limited amount of the available energy is a critical concern [13]. It is important to know the energy consumption limit of a sensor during its active periods, so that it can remain operational over a required lifetime.

Permission to make digital or hard copies of all or part of this work for personal or classroom use is granted without fee provided that copies are not made or distributed for profit or commercial advantage and that copies bear this notice and the full citation on the first page. To copy otherwise, to republish, to post on servers or to redistribute to lists, requires prior specific permission and/or a fee.

ICCAD '06, November 5-9, 2006, San Jose, CA

Copyright 2006 ACM 1-59593-389-1/06/0011 ...\$5.00.
For example, assume that a sensor node must service a sequence of $N$ sensing tasks, and let $t_{j}$ and $d_{j}$ denote the start time and duration of task $j$, where $j=1,2, \ldots, N$. The sensor must survive till the end of the last task, i.e., the required lifetime is $\left(t_{N}+d_{N}\right)$. Let $E_{j}$ denote the energy budget of task $j$. For each task, the sensor energy consumption during task execution should not exceed the budget; otherwise, the sensor's survival is not guaranteed. If the sensor is regulated by a DC-DC converter with power efficiency $\eta_{c}$, then

$$
E_{j}=\eta_{c} \cdot d_{j} \cdot I_{j} \cdot V_{j},
$$

where $I_{j}$ is the battery current, and $V_{j}$ is the battery voltage. Thus, computing the sensor energy budget is reduced to computing the battery current and voltage.

For the sake of simplicity, let $I_{j}$ be constant in the time interval $\left[t_{j}, t_{j}+d_{j}\right]$ corresponding to task $j$, and let $V_{j}\left(t^{*}\right)$ denote the voltage value at time $t^{*} \in\left[t_{j}, t_{j}+d_{j}\right]$. Then, the energy budget $E_{j}$ is bounded as follows:

$$
\begin{aligned}
& \eta_{c} \cdot d_{j} \cdot I_{j} \cdot \min \left\{V_{j}\left(t^{*}\right) \mid t^{*} \in\left[t_{j}, t_{j}+d_{j}\right]\right\}=E_{j}^{-} \leq E_{j}, \\
& \eta_{c} \cdot d_{j} \cdot I_{j} \cdot \max \left\{V_{j}\left(t^{*}\right) \mid t^{*} \in\left[t_{j}, t_{j}+d_{j}\right]\right\}=E_{j}^{+} \geq E_{j} .
\end{aligned}
$$

Symbols $E_{j}^{-}$and $E_{j}^{+}$in Equation (2) denote, respectively, the lower bound and the upper bound on the energy budget of task $j$.

\subsection{Battery Nonlinearities}

Figure 1 shows two measured voltage curves for an Itsy LiIon battery [4] discharged at the constant rates of $222 \mathrm{~mA}$ and $814 \mathrm{~mA}$. Note that the battery voltage changes nonlinearly over time, even in the simplest case of a constantcurrent discharge. Also, the total delivered charge, or the battery capacity, at $814 \mathrm{~mA}$ is less than the charge delivered at $222 \mathrm{~mA}$ before reaching the $3.0-\mathrm{V}$ cutoff. These two phenomena, nonlinear battery voltage changes over time and capacity loss at high discharge rates, must be taken into account during energy budget estimation. The latter effect may especially be important for sensors drawing relatively large currents from the battery during wireless data communication.

When an active interval (discharge) is followed by an idle interval (rest), one can observe a so-called charge recovery effect: the battery voltage increases during that idle interval. In other words, the voltage does not decrease monotonically in general case. Sensors with low duty cycle and relatively high currents during active periods may benefit from charge recovery [8].

Another phenomenon to be considered is capacity fade over time - a Li-Ion battery delivers increasingly less ca- 


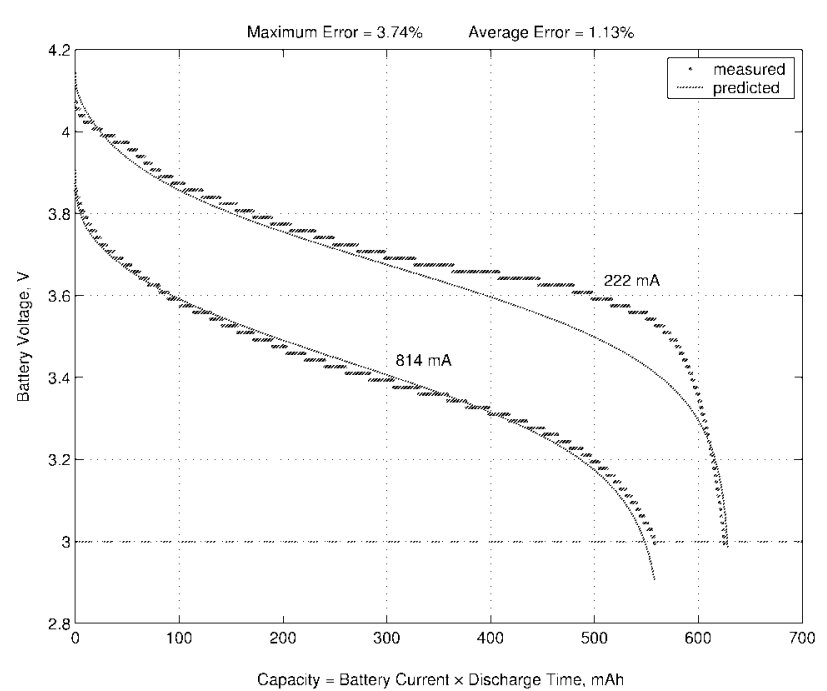

Figure 1: Battery voltage under constant load
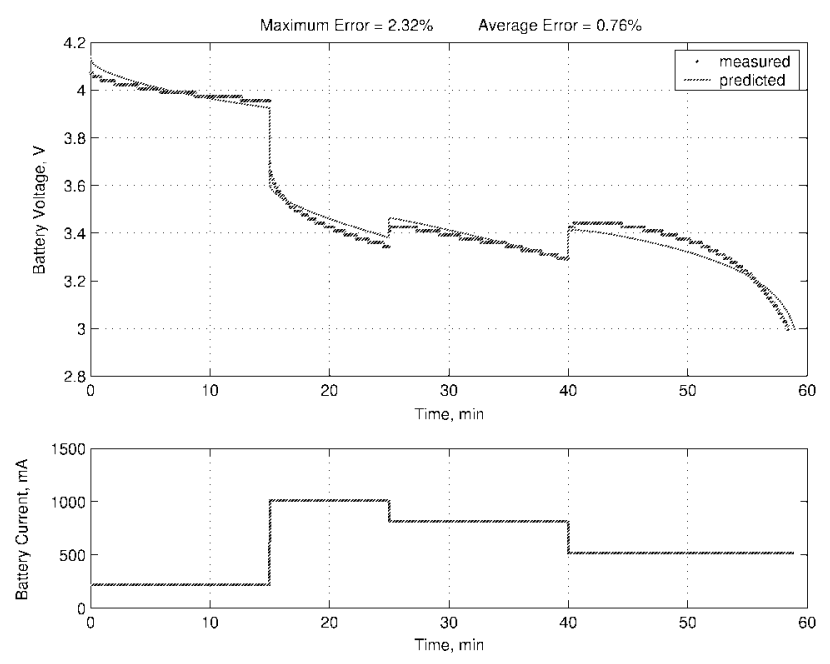

Figure 2: Battery voltage under varying load

pacity as it ages. For example, after 800 charge-discharge cycles at room temperature, a Sony 18650 battery was able to deliver only $70 \%$ of its original capacity [10]. Capacity fade is particularly important in sensors with long lifetimes after deployment.

Computations of the energy budget bounds using Equation (2) require a model that can predict battery voltage changes over time while taking into account high-rate losses, charge recovery, and capacity fading. Such a model has been proposed in [9], and it will be used in this work. The model is reasonably accurate, as indicated by Figures 1-2 showing voltage predictions for constant-current and varying-current examples. The model is described in Section 3, and its parameters are given in Section 7 .

\subsection{Objectives and Contributions}

Given the battery model and the task schedule, our first objective is to obtain the lower bound $E_{j}^{-} \leq E_{j}$ for each task $j=1,2, \ldots, N$. These lower bounds must guarantee

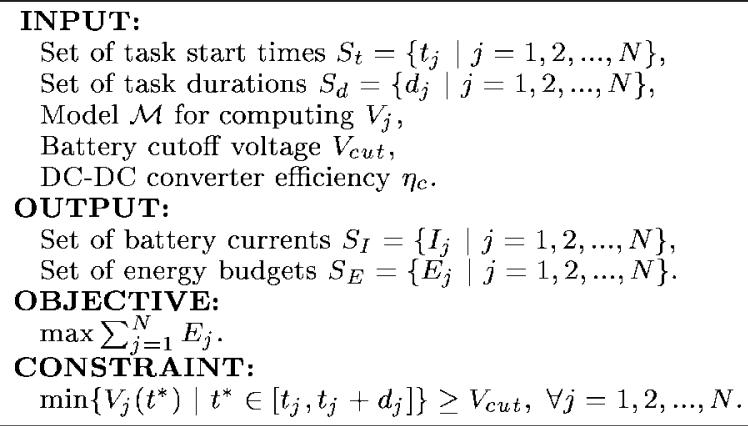

Figure 3: Energy budgeting problem.

sensor's survival till $\left(t_{N}+d_{N}\right)$, i.e., till the end of the last task $N$. We take the lower bound $E_{j}^{-}$as a pessimistic estimate of the energy budget $E_{j}$ for task $j$. Note that a greater energy budget for task $j$ implies that more work can be completed during the time interval $\left[t_{j}, t_{j}+d_{j}\right]$. It is desirable to maximize the energy budgets for all tasks in the sensor's schedule.

Our second objective is to obtain the upper bound $E_{j}^{+} \geq$ $E_{j}$ for comparison purposes. Ideally, the distance between the lower and upper bounds should be small, indicating that our estimate of the energy budget (the lower bound) is not overly pessimistic.

This paper presents a method for computing the energy budget bounds $\left[E_{j}^{-}, E_{j}^{+}\right]$for each task $j$. The proposed method uses the battery voltage estimates provided by the analytical model reported in [9]. This model is computationally intensive - to improve its time and space complexity we also propose alternative approximations for more efficient battery voltage calculations. Thus, our contributions are two-fold: (1) the method for energy budget estimation, and (2) the efficient approximations for battery voltage calculations.

To the best of our knowledge, this is the first work where battery voltage nonlinearities are taken into account during energy budgeting. Related research $[3,1,5,2,12]$ considered charge recovery and high-rate losses, but not capacity fading or battery voltage changes over time. A general overview of battery models and their applications can be found in [11].

\section{ENERGY BUDGETING PROBLEM}

The problem of computing the energy budget can be formulated as shown in Figure 3. Given the task schedule (i.e., $S_{t}$ and $S_{d}$ ), we must first compute task currents $S_{I}$ such that the battery voltage always remains above the cutoff value. Then, we can use Equation (2) to bound the energy budget for each task. By taking the lower bounds as the pessimistic energy budget estimates $S_{E}$, we ensure that all tasks will be serviced. There may be many possible combinations of battery currents $S_{I}$ resulting in many possible energy budgets $S_{E}$. The objective is to select a solution that maximizes the total sum of budgets over all tasks. Once the energy budgets of all tasks are known, a smart sensor can adjust its configuration to meet those budgets on a task-by-task basis.

In this work we consider a special case assuming that the current is the same for all tasks, i.e., $I_{j}=I, \forall j=$ $1,2, \ldots, N$. This restriction considerably reduces the problem complexity. An example of this special case is a periodic 


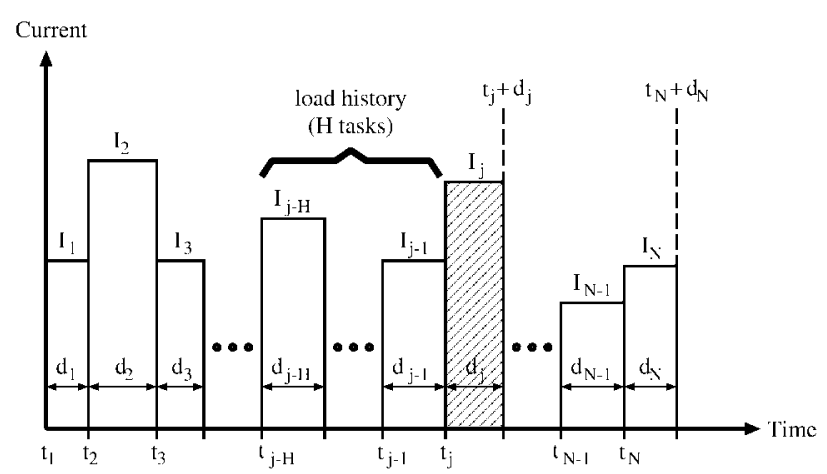

Figure 4: Example of a task schedule

task schedule shown in Figure 5 ( $T_{\text {active }}$ and $T_{\text {idle }}$, however, need not be the same for all tasks).

\section{BATTERY MODEL}

Given a sequence of $N$ battery currents as shown in Figure 4 , model $\mathcal{M}$ computes the battery voltage at time $t^{*} \in$ $\left[t_{j}, t_{j}+d_{j}\right]$ as follows [9]:

$$
\begin{aligned}
& V_{j}\left(t^{*}\right)=V_{0}-r I_{j}- \\
& \quad \phi\left[\left(\gamma_{n}+\gamma_{p}\right) t^{*}+\ln \frac{\alpha_{n}+I_{j} F_{n j}+\sum_{k=1}^{j-1} I_{k} F_{n k}}{\alpha_{p}-I_{j} F_{p j}-\sum_{k=1}^{j} I_{k} F_{p k}}\right],
\end{aligned}
$$

where the $F$-factors are given by

$$
\begin{gathered}
F_{n j}=\frac{e^{-\gamma_{n} t_{j}-e^{-\gamma_{n} t^{*}}}}{\gamma_{n}}+S_{n j}, \\
F_{n k}=\frac{e^{-\gamma_{n} t_{k}-e^{-\gamma_{n}\left(t_{k}+d_{k}\right)}}}{\gamma_{n}}+S_{n k}
\end{gathered}
$$

and

$$
\begin{gathered}
F_{p j}=\frac{e^{\gamma_{p} t^{*}}-e^{\gamma_{p} t_{j}}}{\gamma_{p}}+S_{p j}, \\
F_{p k}=\frac{e^{\gamma_{p}\left(t_{k}+d_{k}\right)}-e^{\gamma_{p} t_{k}}}{\gamma_{p}}+S_{p k} .
\end{gathered}
$$

The functional $S$-series in Equations (5)-(4) are defined in Table 1 . There are nine battery parameters of interest: $V_{0}$, $r, \phi, \alpha_{n}, \alpha_{p}, \beta_{n}, \beta_{p}, \gamma_{n}$, and $\gamma_{p}$ - their detailed description can be found in [9]. Briefly, $\alpha$ quantifies battery capacity, $\beta$ quantifies high-rate losses, and $\gamma$ quantifies capacity fading.

If $\beta_{n, p} \rightarrow \infty$ (no high-rate losses), then each $S$-series evaluates to zero:

$$
\begin{aligned}
& \left.V_{j}\left(t^{*}\right)\right|_{\beta_{n, p} \rightarrow \infty}=V_{0}-r I_{j}-\phi\left[\left(\gamma_{n}+\gamma_{p}\right) t^{*}+\right.
\end{aligned}
$$

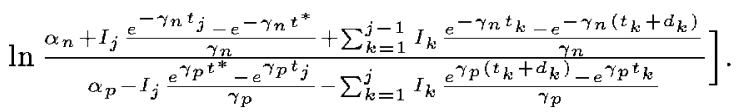

On the other hand, if $\gamma_{n, p} \rightarrow 0$ (no capacity fading), then the battery model is reduced to the following form:

$$
\begin{aligned}
& \left.V_{j}\left(t^{*}\right)\right|_{\gamma_{n, p} \rightarrow 0}=V_{0}-r I_{j}- \\
& \quad \phi \ln \frac{\alpha_{n}+I_{j}\left(t^{*}-t_{j}+S_{n j}\right)+\sum_{k=1}^{j-1} I_{k}\left(d_{k}+S_{n k}\right)}{\alpha_{p}-I_{j}\left(t^{*}-t_{j}+S_{p j}\right)-\sum_{k=1}^{j} I_{k}\left(d_{k}+S_{p k}\right)} .
\end{aligned}
$$

If there are no high-rate losses and no capacity fading (ideal battery), then:

$$
\left.V_{j}\left(t^{*}\right)\right|_{i d e a l}=V_{0}-r I_{j}-\phi \ln \frac{\alpha_{n}+I_{j}\left(t^{*}-t_{j}\right)+\sum_{k=1}^{j-1} I_{k} d_{k}}{\alpha_{p}-I_{j}\left(t^{*}-t_{j}\right)-\sum_{k=1}^{j} I_{k} d_{k}} .
$$

\section{BATTERY VOLTAGE COMPUTATION}

Our battery model $\mathcal{M}$ is relatively complex to be used directly. One disadvantage is the presence of the $S$-series (infinite functional sums). Another disadvantage is that we must keep the history of all previous tasks in order to calculate $\sum_{k=1}^{j-1} I_{k} F_{n k}$ and $\sum_{k=1}^{j-1} I_{k} F_{p k}$. These two sums must be recomputed for each $j=1,2, \ldots, N$. For any given task $j$, the sums may be recomputed several times, since a user may check several time instances $t^{*} \in\left[t_{j}, t_{j}+d_{j}\right]$, reevaluating the $S$-series accordingly. Let $X$ denote the time complexity of evaluation of the $S$-series, and let $Y$ denote the maximum number of checked $t^{*}$ per task. Then, the resulting time complexity is $O\left(N^{2} X Y\right)$. The space complexity is $O(N)$, since the entire load history must be stored.

To speed up computations and reduce storage requirements, we propose alternative approximations that avoid using the series and the entire load history. These approximations are, in fact, the lower and upper bounds, $V^{-}$and $V^{+}$, for the battery voltage computed by model $\mathcal{M}$.

The bounding approximations for $V_{j}\left(t^{*}\right)$ are as follows:

$$
\begin{aligned}
& V_{j}^{-}\left(t^{*}\right)=V_{0}-r I_{j}- \\
& \quad \phi\left[\left(\gamma_{n}+\gamma_{p}\right) t^{*}+\ln \frac{\alpha_{n}+I_{j} F_{n j}^{+}+\sum_{k=1}^{j-1} I_{k} F_{n k}^{+}}{\alpha_{p}-I_{j} F_{p j}^{+}-\sum_{k=1}^{j} I_{k} F_{p k}^{+}}\right], \\
& V_{j}^{+}\left(t^{*}\right)=V_{0}-r I_{j}- \\
& \quad \phi\left[\left(\gamma_{n}+\gamma_{p}\right) t^{*}+\ln \frac{\alpha_{n}+I_{j} F_{n j}^{-}+\sum_{k=1}^{j-1} I_{k} F_{n k}^{-}}{\alpha_{p}-I_{j} F_{p j}^{-}-\sum_{k=1}^{j} I_{k} F_{p k}^{-}}\right] .
\end{aligned}
$$

These approximations use the bounds for the $F$-factors, which are based on the respective bounds for the $S$-series (see Table 1). ${ }^{1}$ For example, when computing $F_{n k}^{+}$we use Equation (4) with $S_{n k}^{+}$.

In Table 1 one can see that the infinite sums (functional series) are replaced by $M$-term sums where necessary. Also, at most $H$ previous tasks are considered, instead of the entire load history. Hence, the corresponding time and space complexities become $O(N H M Y)$ and $O(H)$, respectively. The values of $M$ and $H$ are a user's choice - greater values will yield better approximations, but will require more computational time and space.

\section{BATTERY CURRENT COMPUTATION}

Once the battery voltage reaches the cutoff value $V_{c u t}$, the sensor is no longer operational. Recall that, for the sake of simplicity, we assumed that $I_{j}=I, \forall j=1,2, \ldots, N$. Our objective is to maximize the value of $I$, which in turn maximizes the energy budget. To ensure that the sensor survives all tasks, we must guarantee that $V_{j}\left(t^{*}\right) \geq V_{c u t}$ for any $t^{*} \in\left[t_{j}, t_{j}+d_{j}\right], \forall j=1,2, \ldots, N$.

Computation of $V_{j}\left(t^{*}\right)$ using model $\mathcal{M}$, given by Equation (3), is expensive. Instead we will use the lower bound $V_{j}^{-}\left(t^{*}\right) \leq V_{j}\left(t^{*}\right)$ while computing the battery current. To ensure solution feasibility it is sufficient to guarantee that $V_{j}^{-}\left(t^{*}\right) \geq V_{\text {cut }}$.

We perform the following three steps to find $I$ that will be used for computing the task energy budgets.

${ }^{1}$ Derivations of the upper and lower bounds for $S_{n k}$ and $S_{p k}$ from Table 1 are omitted due to the lack of space. 


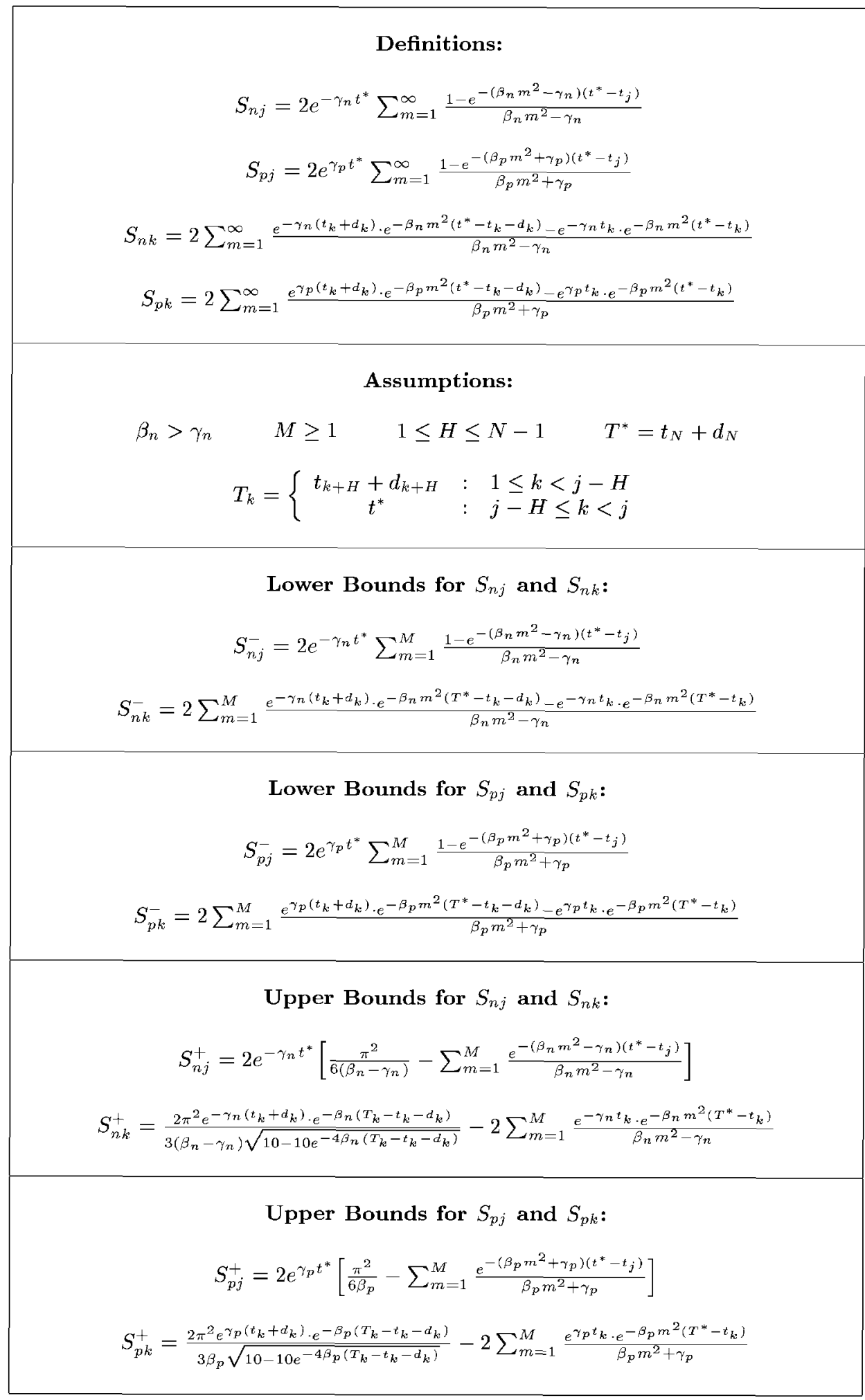

Table 1: Definitions and bounds for $S$-series. 
Step 1: As $V_{N}\left(t_{N}+d_{N}\right) \geq V_{N}^{-}\left(t_{N}+d_{N}\right)$, we safely let

$$
\begin{aligned}
& V_{c u t}=V_{N}^{-}\left(t_{N}+d_{N}\right)=V_{0}-r I- \\
& \quad \phi\left[\left(\gamma_{n}+\gamma_{p}\right)\left(t_{N}+d_{N}\right)+\ln \frac{\alpha_{n}+I F_{n N}^{+}+\sum_{k=1}^{N-1} I F_{n k}^{+}}{\alpha_{p}-I F_{p N}^{+}-I \sum_{k=1}^{N-1} I F_{p k}^{+}}\right] .
\end{aligned}
$$

Note that $t^{*}=t_{N}+d_{N}$. Equation (11) has only one unknown, current $I$ - its value can be found numerically. Since Expression (11) is monotonic in $I$, we can use a fast binary search to find the unknown current value.

Step 2: As $V_{N}\left(t_{N}\right) \geq V_{N}^{-}\left(t_{N}\right)$, it is sufficient to test whether $V_{N}^{-}\left(t_{N}\right) \geq V_{\text {cut }}$ given the current value $I$ found in Step 1. If the test fails, i.e., $V_{N}^{-}\left(t_{N}\right)<V_{c u t}$, we must decrease $I$ so that

$$
\begin{aligned}
& V_{c u t}=V_{N}^{-}\left(t_{N}\right)=V_{0}-r I- \\
& \quad \phi\left[\left(\gamma_{n}+\gamma_{p}\right) t_{N}+\ln \frac{\alpha_{n}+\sum_{k=1}^{N-1} I F_{n k}^{+}}{\alpha_{p}-I \sum_{k=1}^{N-1} I F_{p k}^{+}}\right] .
\end{aligned}
$$

In other words, unless $V_{N}^{-}\left(t_{N}\right) \geq V_{c u t}$, we must perform another binary search for a smaller battery current $I$ using Expression (12). Note that $t^{*}=t_{N}$, and according to Equations (4)-(5), $F_{n N}=F_{p N}=0$ (from Table $1, S_{n N}=S_{p N}=0$ if $\left.t^{*}=t_{N}\right)$.

Step 3: In the previous two steps, we checked the contraint $V_{N}\left(t^{*}\right) \geq V_{c u t}$ for $t^{*}=t_{N}$ and $t^{*}=t_{N}+d_{N}$. Strictly speaking, we should also check other times instances $t^{*} \in$ $\left(t_{N}, t_{N}+d_{N}\right)$. Based on our numerous observations, we conjecture that if the voltage is above the cutoff value at the time boundaries of a constant-current task, then it is above the cutoff value anywhere between those boundaries. In other words, if for some task $j$ we have $V_{j}\left(t_{j}\right) \geq V_{c u t}$ and $V_{j}\left(t_{j}+d_{j}\right) \geq V_{c u t}$, then checking whether $V_{j}\left(t^{*}\right) \geq V_{c u t}$ for $t^{*} \in\left(t_{j}, t_{j}+d_{j}\right)$ is not necessary. However, this conjecture has not been proven formally. Consequently, this potentially redundant step is included in the algorithm. We first partition the time interval $\left(t_{N}, t_{N}+d_{N}\right)$ into $Y$ subintervals of equal length $\Delta$. Then, for each $y=1,2, \ldots, Y$, we let $t^{*}=t_{N}+y \Delta$ and check whether $V_{N}^{-}\left(t^{*}\right) \geq V_{c u t}$. If the test fails, we decrease $I$ so that $V_{N}^{-}\left(t^{*}\right)=V_{c u t}$.

In general, considering only the last task $j=N$ is not sufficient - recall that it is sometimes possible for recovery effects to mask constraint violations of the earlier tasks. To ensure solution feasibility, we repeat the above three steps for all tasks in reverse order, reducing the value of $I$ as needed. In practice, however, it is very unlikely that task $N$ survives, while one of the earlier tasks $j=N-1, N-2, \ldots, 1$ has failed. In other words, the original value of $I$ found using Expression (11) will rarely be recomputed.

Note that the current $I$ computed based on $V_{j}^{-}=V_{\text {cut }}$ (i.e., using our approximations) will be less than the optimal $I_{\max }$ computed based on $V_{j}=V_{c u t}$ (i.e., using our model $\mathcal{M}$ directly). However, since our approximations require less computational time and space than $\mathcal{M}$, the complexity of computing $I$ will be lower than the complexity of computing $I_{\max }$.

\section{ENERGY BUDGET COMPUTATIONS}

Once current $I$ is known, we compute the lower bound $E_{j}^{-}$ and the upper bound $E_{j}^{+}$of the energy budget as follows:

$$
\begin{aligned}
& E_{j}^{-}=\eta_{c} \cdot d_{j} \cdot I \cdot \min \left\{V_{j}^{-}\left(t^{*}\right) \mid t^{*} \in\left[t_{j}, t_{j}+d_{j}\right]\right\} \\
& E_{j}^{+}=\eta_{c} \cdot d_{j} \cdot I \cdot \max \left\{V_{j}^{+}\left(t^{*}\right) \mid t^{*} \in\left[t_{j}, t_{j}+d_{j}\right]\right\}
\end{aligned}
$$

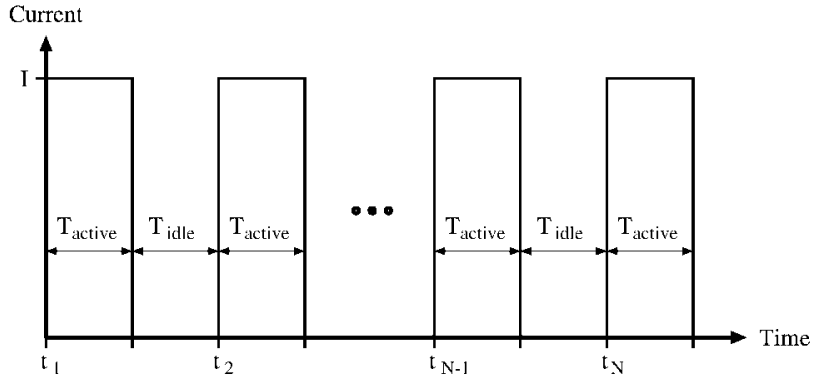

Figure 5: Example of a sensor load.

\begin{tabular}{|c||c|c|c|c|c|c|}
\hline S. & $N$ & $\begin{array}{c}T_{\text {active, }} \\
\text { min }\end{array}$ & $\begin{array}{c}T_{\text {idle, }} \\
\text { min }\end{array}$ & $\begin{array}{c}\text { Total, } \\
\mathrm{h}\end{array}$ & $\begin{array}{c}\text { Active, } \\
\mathrm{h}\end{array}$ & $\begin{array}{c}\text { Duty } \\
\text { Cycle }\end{array}$ \\
\hline \hline S1 & 3000 & 1 & 99 & 5000 & 50 & 0.01 \\
\hline S2 & 3000 & 1 & 9 & 500 & 50 & 0.1 \\
\hline S3 & 3000 & 0.1 & 0.9 & 50 & 5 & 0.1 \\
\hline S4 & 300 & 0.1 & 9.9 & 50 & 0.5 & 0.01 \\
\hline S5 & 300 & 0.1 & 0.9 & 5 & 0.5 & 0.1 \\
\hline
\end{tabular}

Table 2: Five scenarios under consideration.

Again, instead of using our model $\mathcal{M}$ to compute $V_{j}$ directly, we have used our approximations $V_{j}^{-}$and $V_{j}^{+}$in order to speed up computations. This computational speedup comes at the expense of solution optimality, since we let $E_{j}=E_{j}^{-}$, and since $V_{j}^{-} \leq V_{j} \Rightarrow I \leq I_{\max }$. However, the use of $V_{j}^{-}$instead of $V_{j}$ while computing the battery currents and energy budgets is optional, i.e., it is a user's choice to trade off solution optimality for computational complexity.

If our conjecture (see Section 5, Step 3) is true, then the lower bound $E_{j}^{-}$can be computed using a simpler equation:

$$
E_{j}^{-}=\eta_{c} \cdot d_{j} \cdot I \cdot \min \left\{V_{j}^{-}\left(t_{j}\right), V_{j}^{-}\left(t_{j}+d_{j}\right)\right\}
$$

Recall that we use the lower bound $E_{j}^{-}$as the energy budget, and compare it against the upper bound $E_{j}^{+}$. If the difference between these two bounds is small, then the energy budget is not overly pessimistic. Since the upper bound $E_{j}^{+}$is used only for comparison purposes, its accuracy is not critical. It can be computed using a simpler equation:

$$
E_{j}^{+}=\eta_{c} \cdot d_{j} \cdot I \cdot \max \left\{V_{j}^{+}\left(t_{j}\right), V_{j}^{+}\left(t_{j}+d_{j}\right)\right\}
$$

\section{EVALUATION RESULTS}

To illustrate the use of the proposed energy budgeting method we consider an example of a periodic sensor load shown in Figure 5. A sensor is activated $N$ times: during each period it is active for $T_{\text {active }}$ minutes and idle for $T_{\text {idle }}$ minutes. We study five sensing scenarios shown in Table 2, letting the sensor lifetime range from 5 hours to 5000 hours.

For each scenario, we consider three battery variants shown in Table 3. For all three batteries, $V_{0}=3.76 \mathrm{~V}, r=400 \mathrm{~m} \Omega$, and $\phi=0.125 \mathrm{~V}$. For battery B1, we further let $\alpha_{n}=15$ $\mathrm{mAh}, \alpha_{p}=655 \mathrm{mAh}, \beta_{n}=2.501 / \mathrm{min}$, and $\beta_{p}=0.50$ $1 / \mathrm{min}$. These values were used to predict measured battery voltages shown in Figures 1-2. Also, we let both $\gamma_{n}$ and $\gamma_{p}$ equal $0.0000171 / \mathrm{min}$. These values were obtained by fitting the cycling fade model from [9] to the experimental 


\begin{tabular}{|c||c|c||c|c||c|c|}
\hline B. & $\begin{array}{c}\alpha_{n} \\
\mathrm{mAh}\end{array}$ & $\begin{array}{c}\alpha_{p}, \\
\mathrm{mAh}\end{array}$ & $\begin{array}{c}\beta_{n}, \\
1 / \mathrm{min}\end{array}$ & $\begin{array}{c}\beta_{p}, \\
1 / \mathrm{min}\end{array}$ & $\begin{array}{c}\gamma_{n}, \\
1 / \mathrm{min}\end{array}$ & $\begin{array}{c}\gamma_{p}, \\
1 / \mathrm{min}\end{array}$ \\
\hline \hline B1 & 15 & 655 & 2.50 & 0.50 & 0.000017 & 0.000017 \\
\hline B2 & 15 & 655 & 1.25 & 0.25 & 0.000034 & 0.000034 \\
\hline B3 & 15 & 655 & $\infty$ & $\infty$ & 0 & 0 \\
\hline
\end{tabular}

Table 3: Three batteries under consideration.

data from $[10]$ for a Sony battery at the room temperature (the maximum error was $3 \%$ ).

Battery $\mathrm{B} 2$ is derived from $\mathrm{B} 1$ by halving $\beta_{n, p}$ and doubling $\gamma_{n, p}$, which makes B2 more lossy than B1. Battery B3 represents an ideal case.

For our approximations we let $M=H=10$. Also, we used Equations (14)-(15) while computing $E_{j}^{-}$and $E_{j}^{+}$.

\subsection{Performance of Voltage Approximations}

There are two contributors to the difference between the lower and the upper bounds of the energy budget. First is the task duration and current - a longer tasks with a higher current will result in a greater difference between $V_{j}\left(t_{j}\right)$ and $V_{j}\left(t_{j}+d_{j}\right)$, which leads to a greater difference between the energy budget bounds $E_{j}^{+}$and $E_{j}^{-}$. This first contributor is specific to the task characteristics, not voltage approximations. Recall that battery B3 requires no approximations, yet the energy budget bounds do not match - the greater the task charge $\left(I \cdot T_{\text {active }}\right)$, the greater the difference between $E_{j}^{+}$and $E_{j}^{-}$. For example, scenarios S4 and S5 have the highest charge consumption per task (7.12 Coulombs), and consequently, the worst maximum difference of $0.42 \%$ among the cases involving battery B3.

The second contributor is the quality of the proposed voltage approximations that can be seen in ten cases involving batteries $\mathrm{B} 1$ and $\mathrm{B} 2$. In six of those cases, the bound difference is under $5 \%$, which implies that our approximations were somewhat accurate in comparison with model $\mathcal{M}$ given by Equation (3). In other words, if we were to use battery voltage model $\mathcal{M}$ directly, our exact results would match approximated solutions within $5 \%$. The other four cases did not perform well - in two of them (S3-B2 and S5-B2) even the average error exceeded $5 \%$. Such a poor performance is due to the overly pessimistic lower bound. ${ }^{2}$ The use of our approximations is not justified in those cases, unless the complexity of model $\mathcal{M}$ is an important concern.

\subsection{Performance of Batteries}

The battery voltage is usually lower for later tasks, so the energy budget is reduced accordingly. For example, in case S3-B3 the budget of the first task is $3.27 \mathrm{~J}$, while the budget for the last task is $2.36 \mathrm{~J}$, or $28 \%$ less. Smart sensors must budget their energy consumption accordingly, compensating for changes in the battery voltage.

Cases S1-B1 and S1-B2 illustrate the effect of capacity fading over time. Compared to the 13.0-mA current for the ideal battery B3, the battery current is only $9.9 \mathrm{~mA}$ for B1 (24\% less) and $7.4 \mathrm{~mA}$ for B2 (43\% less). The task energy budgets (lower bounds) for these three cases are shown in Figure 6. Note that scenario S1 corresponds to the longest sensor lifetime of $5000 \mathrm{~h}$, and capacity fading can no longer

\footnotetext{
${ }^{2}$ The output produced by model $\mathcal{M}$ was much closer to the
} upper bound than to the lower bound.

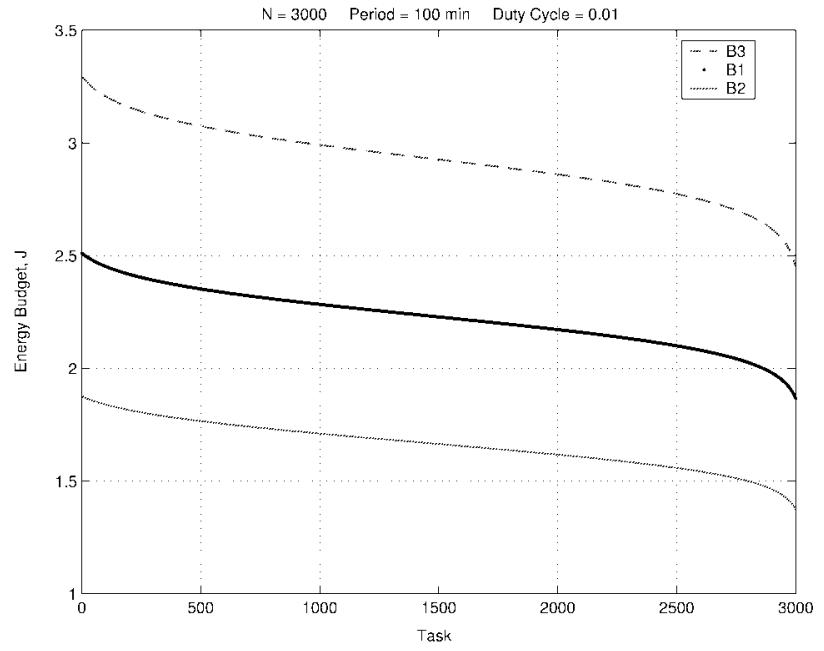

Figure 6: Task Energy Budgets for Scenario S1.

be ignored. Relatively short lifetimes of the other scenarios do not expose fading effects.

Shorter sensing times usually lead to higher allowable discharge currents. For example, the sensing time of scenarios $\mathrm{S} 1$ and $\mathrm{S} 2$ is $50 \mathrm{~h}$, while the sensing time of scenarios S4 and $\mathrm{S} 5$ is only $0.5 \mathrm{~h}$. This leads to a nearly 100 -fold current difference. Due to high-rate losses, the latter scenarios need special consideration. Even though scenarios S4 and S5 have the same sensing time, S4 performs better than S5 because of the 11-fold difference in $T_{\text {idle }}(9.9 \mathrm{~min}$ for $\mathrm{S} 4$ versus 0.9 min for S5). Longer idle intervals allow for greater charge recovery that mitigates high-rate losses. In other words, capacity loss due to high battery currents can be lessened by enforcing very low duty cycles.

Not surprisingly, battery B2 performed worse than B1 (less lossy), i.e., the energy budgets in cases involving B2 are worse than those involving B1. Also, note that the bound differences in cases $\mathrm{S} 3-\mathrm{B} 2$ and $\mathrm{S} 5-\mathrm{B} 2$ are worse than those in cases S3-B1 and S5-B1. Ideal battery B3 is a clear winner in Table 4 , as it has no losses and requires no approximations.

\subsection{General Recommendations}

Time and space savings due to the lower complexity of the approximations may be particularly important when energy budgeting is performed by a sensor itself, e.g., in response to the change in a sensing scenario or a battery. However, the accuracy loss may exceed the acceptable error margins, which may lead to overly pessimistic energy budgets. In such cases, which are identifiable by a large difference between the lower and upper bounds, a user should work directly with the battery voltage model $\mathcal{M}$ despite its complexity. The proposed methods for bounding voltages and energy budgets are likely to perform better for:

- batteries with larger $\beta_{n, p}$ (less high-rate losses),

- batteries with smaller $\gamma_{n, p}$ (less capacity fading),

- scenarios with shorter $T_{\text {active, }}$

- scenarios with longer $T_{\text {idle }}$.

Last, we point out that the quality of energy budgeting depends on the quality of the battery model. Since no model perfectly matches reality, the computed energy budget values should be used with reasonable caution. 


\begin{tabular}{|c|c||c|c||c|c||c|c|}
\hline \multicolumn{2}{|c||}{ Case } & \multicolumn{2}{c||}{ First Task } & \multicolumn{2}{c||}{ Last Task } & $\frac{E_{j}^{+}-E_{j}^{-}}{E_{j}^{-}} \mid j=1,2, \ldots, N$ \\
\hline $\begin{array}{c}\text { Scenario- } \\
\text { Battery }\end{array}$ & $\begin{array}{c}\text { Current } \\
I, \mathrm{~mA}\end{array}$ & $\begin{array}{c}\text { Lower Bound } \\
E_{1}^{-}, \mathrm{J}\end{array}$ & $\begin{array}{c}\text { Upper Bound } \\
E_{1}^{+}, \mathrm{J}\end{array}$ & $\begin{array}{c}\text { Lower Bound } \\
E_{N}^{-}, \mathrm{J}\end{array}$ & $\begin{array}{c}\text { Upper Bound } \\
E_{N}^{+}, \mathrm{J}\end{array}$ & $\begin{array}{c}\text { Maximum } \\
\%\end{array}$ & $\begin{array}{c}\text { Average } \\
\%\end{array}$ \\
\hline \hline S1-B1 & 9.9 & 2.51 & 2.51 & 1.87 & 1.88 & 0.62 & 0.03 \\
\hline S1-B2 & 7.4 & 1.88 & 1.88 & 1.38 & 1.39 & 0.89 & 0.04 \\
\hline S1-B3 & 13.0 & 3.30 & 3.30 & 2.45 & 2.46 & 0.17 & 0.01 \\
\hline \hline S2-B1 & 12.7 & 3.22 & 3.22 & 2.33 & 2.36 & 1.45 & 0.04 \\
\hline S2-B2 & 12.3 & 3.12 & 3.12 & 2.33 & 2.35 & 0.99 & 0.05 \\
\hline S2-B3 & 13.0 & 3.30 & 3.30 & 2.45 & 2.46 & 0.17 & 0.01 \\
\hline \hline S3-B1 & 126.9 & 3.17 & 3.18 & 2.29 & 2.48 & 8.60 & 1.07 \\
\hline S3-B2 & 115.3 & 2.88 & 2.90 & 2.08 & 2.29 & 14.92 & 7.28 \\
\hline S3-B3 & 130.5 & 3.27 & 3.27 & 2.36 & 2.36 & 0.35 & 0.01 \\
\hline S4-B1 & 1142.5 & 25.29 & 25.89 & 20.57 & 20.98 & 2.33 & 0.76 \\
\hline S4-B2 & 1105.1 & 24.41 & 25.13 & 19.89 & 20.56 & 3.82 & 1.24 \\
\hline S4-B3 & 1186.1 & 26.63 & 26.74 & 21.35 & 21.38 & 0.42 & 0.08 \\
\hline S5-B1 & 990.3 & 22.33 & 22.79 & 17.83 & 19.11 & 7.19 & 2.74 \\
\hline S5-B2 & 609.1 & 14.32 & 14.58 & 10.96 & 12.88 & 17.50 & 8.33 \\
\hline S5-B3 & 1186.1 & 26.63 & 26.74 & 21.35 & 21.38 & 0.42 & 0.08 \\
\hline
\end{tabular}

Table 4: Energy budget bounds for the first and last tasks and relative bound difference over all tasks.

\section{CONCLUSION}

Battery-powered wireless sensors are severely constrained by the amount of the available energy. This paper described a method for computing the lower and upper bounds for the energy budget per task for sensors that must survive for a specified period of time. We assumed that the sensor's task schedule was known and each task drew the same current from the battery.

The proposed bounding method used an analytical model for predicting the battery voltage given the discharge current, while taking into account battery capacity loss at high discharge rates, charge recovery, and capacity fade over time. Since it is computationally expensive to use this model directly, we also proposed alternative approximations for more efficient voltage calculations.

We considered three variants of a Li-ion battery and five sensing scenarios, resulting in 15 different cases. The computed energy budget bounds were within $5 \%$ of each other in 11 cases. Our future effort will target better voltage approximations and less restrictive assumptions, e.g., allowing for varying battery current across tasks.

\section{REFERENCES}

[1] J. Ahmed and C. Chakrabarti. A dynamic task scheduling algorithm for battery powered dvs systems. In Proc. International Conference on Circuits and Systems, pages 813-816. IEEE, May 2004.

[2] Y. Cai, S. Reddy, I. Pomeranz, and B. Al-Hashimi. Battery-aware dynamic voltage scaling in multiprocessor embedded systems. In Proc. International Conference on Circuits and Systems, pages 616-619. IEEE, May 2005.

[3] S. Dasika, S. Vrudhula, K. Chopra, and R. Srinivasan. A framework for battery-aware sensor management. In Proc. Design, Automation and Test in Europe Conference and Exhibition, pages 962-967. ACM/IEEE, Feb. 2004.
[4] W. Hamburgen, D. Wallach, M. Viredaz, L. Brakmo, C. Waldspurger, J. Bartlett, T. Mann, and K. Farkas. Itsy: Stretching the bounds of mobile computing. IEEE Computer, 34(4):28-37, Apr. 2001.

[5] S. Hong, D. Kim, and J. Kim. Battery-aware real-time task scheduling in wireless sensor networks. In Proc. International Conference on Embedded and Real-Time Computing Systems and Applications, pages 269-272. IEEE, Aug. 2005.

[6] Intel/University of California-Berkeley. Mote Project. http://www.intel.com/research/exploratory/motes.htm, 2006.

[7] Massachusetts Institute of Technology. uAMPS Project. http://mtlweb.mit.edu/researchgroups/icsystems/uamps, 2004.

[8] S. Park, A. Savvides, and M. Srivastava. Battery capacity measurement and analysis using lithium coin cell battery. In Proc. International Symposium on Low Power Electronics and Design, pages 382-387. ACM/IEEE, Aug. 2001.

[9] D. Rakhmatov. Battery voltage prediction for portable systems. In Proc. International Conference on Circuits and Systems, pages 4098-4101. IEEE, May 2005.

[10] P. Ramadass, B. Haran, R. White, and B. Popov. Capacity fade of sony 18650 cells cycled at elevated temperatures. part I: Cycling performance. Journal of Power Sources, 112:606-613, Jan. 2002.

[11] R. Rao, S. Vrudhula, and D. Rakhmatov. Battery modeling for energy-aware system design. IEEE Computer, 36(12):77-87, Dec. 2003.

[12] P. Rong and M. Pedram. Battery-aware power management based on markovian decision processes. IEEE Trans. Computer-Aided Design of Integrated Circuits and Systems, 25(7):1337-1349, July 2006.

[13] B. Sadler. Fundamentals of energy-constrained sensor network systems. IEEE Aerospace and Electronic Systems Magazine, 20(8):17-35, Aug. 2005. 